\title{
LOW ENERGY SOLAR NUCLEAR PARTICLE IRRADIATION OF LUNAR AND METEORITIC BRECCIAS
}

\author{
J. C. DRAN, J. P. DURAUD, and M. MAURETTE \\ Centre de Spectrométrie de Masse du CNRS-9I ORSAY-France
}

\begin{abstract}
We have studied by combined high voltage and scanning electron microscopies both the latent and etched track distributions in lunar fragmental rocks as well as in solar type gas-rich meteorites. We have used a new experimental approach for studying gas-rich meteorites to avoid problems with the interpretation of the results. Some implications of the present results concerning the ancient solar flare cosmic rays and the origin of the track distributions in gas-rich meteorite crystals will be discussed.
\end{abstract}

\section{Introduction}

In 1960, R. M. Walker opened up a completely new field of research on the premise that lunar material should contain nuclear particle tracks produced by energetic heavy nuclei both of solar and galactic origin [1]. In 1963, Suess et al. [2] suggested that the large amount of rare gas found in the dark parts of 'solar' type gas-rich meteorites could be due to an ancient implantation of very low energy solar wind nuclei in their constituent grains, before the compaction of the grains into the meteorite dark parts. Since these 2 original suggestions concerning the possibility of finding natural mineral grains irradiated in solar nuclear particles of various types, a lot of work has been done mainly on lunar material [3], but also on gas-rich meteorites [4, 5, 6] and more recently on Antarctica dust [7], in view of finding such grains and exploiting their solar irradiation records for different purposes, in particular to determine the characteristics of the ancient solar flare cosmic rays as well as to decipher the origin of gas-rich meteorites.

In this paper we will present a new experimental approach for the problem of finding mineral grains 'individually' irradiated in space before their 'compaction' into lunar and meteoritic rocks considered as breccias. In particular we have first defined the microscopic irradiation record in lunar dust grains - which certainly get individually irradiated in solar nuclear particles-by using not only scanning electron microscopy and optical microscopy to study etched tracks but also transmission electron microscopy to look for that part of the high resolution irradiation record not appearing with the previously used other techniques. Then we observed possible changes in this record as the grains get artificially heated or naturally 'sintered' into lunar breccias 10046 and 10059 . Finally with the same methods we searched for grains showing a 'lunar type' solar irradiation record in the dark parts of several gas-rich meteorites (Weston, Pantar I, Breitschied, Kapoyeta).

In the first part of this paper we will point out some factors which could obscure the solar record in gas-rich meteorites. Then we will describe how we minimized these factors not taken into serious consideration before our work by presenting our main 
observations of lunar and meteoritic grains by scanning and transmission electron microscopies. Finally we will discuss the present possibilities in finding extraterrestrial mineral grains with a good solar irradiation record allowing the determination of some characteristics of ancient solar nuclear particle fluxes and we will question the possibility of clearly deciphering the origin of gas-rich meteorites by analyzing their irradiation record.

\section{Experimental Approach}

Before the study of lunar samples and our transmission electron microscope observations of extraterrestrial matter [8,9] it was generally considered that the following criteria [1, 4, 5] should be satisfied in identifying grains individually irradiated in space before their 'compaction' into the meteorites:

(1) a clear enrichment of the most superficial layer of the grains in solar type raregas should be observed;

(2) the grains should contain a high density, $\varrho$, of long nuclear particle tracks well above the maximum contribution $\left(\varrho \lesssim 10^{7}\right.$ tracks $\left.\cdot \mathrm{cm}^{-2}\right)$ expected from the exposure of the meteorite in the $\mathrm{VH}$ nuclei of the galactic cosmic rays;

(3) the track length distribution in the high $\varrho$ grains should correspond to that expected from solar flare VH nuclei in being peaked at about 5 to $10 \mu$;

(4) a 'homogeneous' edge zoned track distribution, characterized by a sudden drop of the track density on a depth of about 10 to $20 \mu$ should be observed in the high $\varrho$ grains; this track gradient would simply reflect the 'attenuation' with the depth into the grains of a composite beam of $\mathrm{VH}$ nuclei containing more low energy nuclei than more energetic ones.

There are several limitations in applying these track criteria:

(1) the high track density criterion alone is only valid if the tracks are long and if an important proportion of the grains (at least a few percent) contain high $\varrho$ values. Otherwise it can also be expected that the tracks have been produced by spallation recoils or that the grains are uranium bearing phases because it is known that in meteoritic [10] and lunar [11] materials spontaneously fissionable elements are indeed very inhomogeneously distributed in minor mineral phases which could then get loaded with high fission track densities in a relatively short period of time;

(2) track length greater than about $1 \mu$ can only be directly measured with an optical microscope and this is not easy when the track density is $>10^{8}$ tracks $\cdot \mathrm{cm}^{-2}$ or when they are found in an edge zoning in the grains; therefore it is difficult to verify if the tracks in the high $\varrho$ grains are those expected from solar flare $\mathrm{VH}$ nuclei;

(3) before using the track zoning criterion which is apparently the easiest to apply one must be sure that the actual external surfaces of the grains are the true 'premeteoritic' surfaces exposed in space and that no effect else than a solar VH beam attenuation with the depth can produce a track gradient.

These last limitations concerning the most frequently used [4, 5, 6] 'fossil track zoning' seem to be the most severe for the following reasons:

(1) by using $1 \mathrm{MeV}$ and $100 \mathrm{keV}$ electron microscopies we observed very frequently 


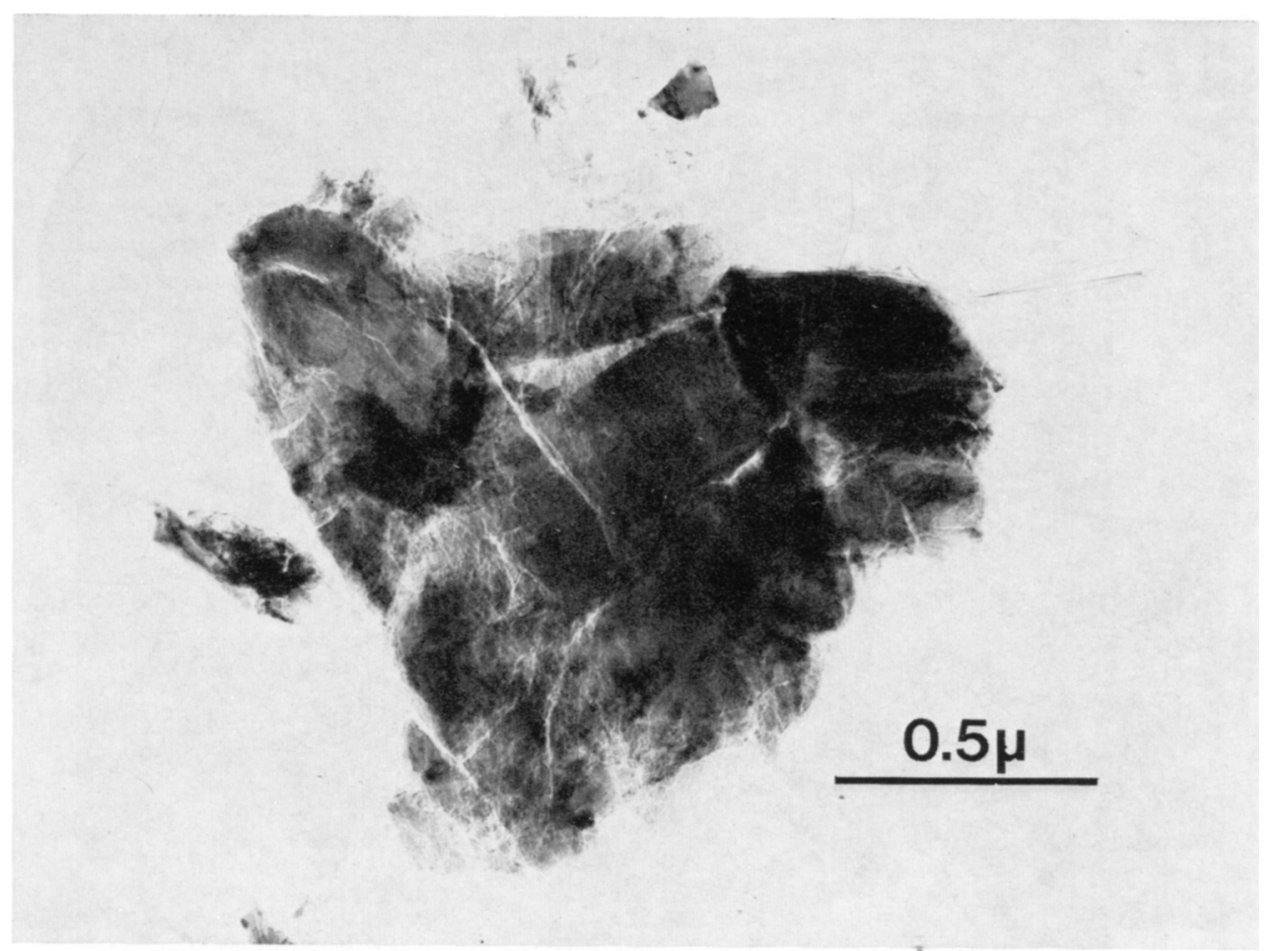

Fig. 1. $100 \mathrm{keV}$ dark field micrograph of a slightly etched pyroxene grain, extracted from the dark part of the Kapoyeta meteorite and showing the extensive microfracturing in the grain.

an extensive microfracturing (Figure 1) in the grains extracted from the dark parts of several gas-rich meteorites. Therefore for track zoning studies we decided to drop the 'hand picking' techniques previously applied $[4,5,6]$ because it is likely that during such an extraction the highly fractured grains would lost parts from their external surfaces. Instead we applied to lunar and meteoritic breccias a technique already developped for lunar igneous rocks [11] and where a polished section of a chunk of meteorite was first prepared by using epoxy resin; then the section was etched to reveal the fossil tracks and observed with a scanning electron microscope (SEM) with results similar to those reported in Figure 2 for a dark part fragment* of Kapoyeta; this etched section shows an extensive grain fracturing which again raises serious doubts about the validity of any 'hand picking' technique either to preserve the external surface of the grains or to search for grains with 'rounded' habits indicative of their 'premeteoritic' erosion. Furthermore, even by using this technique several small grains were still removed from the surface after the etching. Therefore we decided to etch a crushed residue from a dark part and to search for latent and slightly etched

* Another interesting advantage of this technique consists in the possibility of analysing in great detail the boundary between a dark and a light part, by polishing a fragment containing these 2 different parts. 


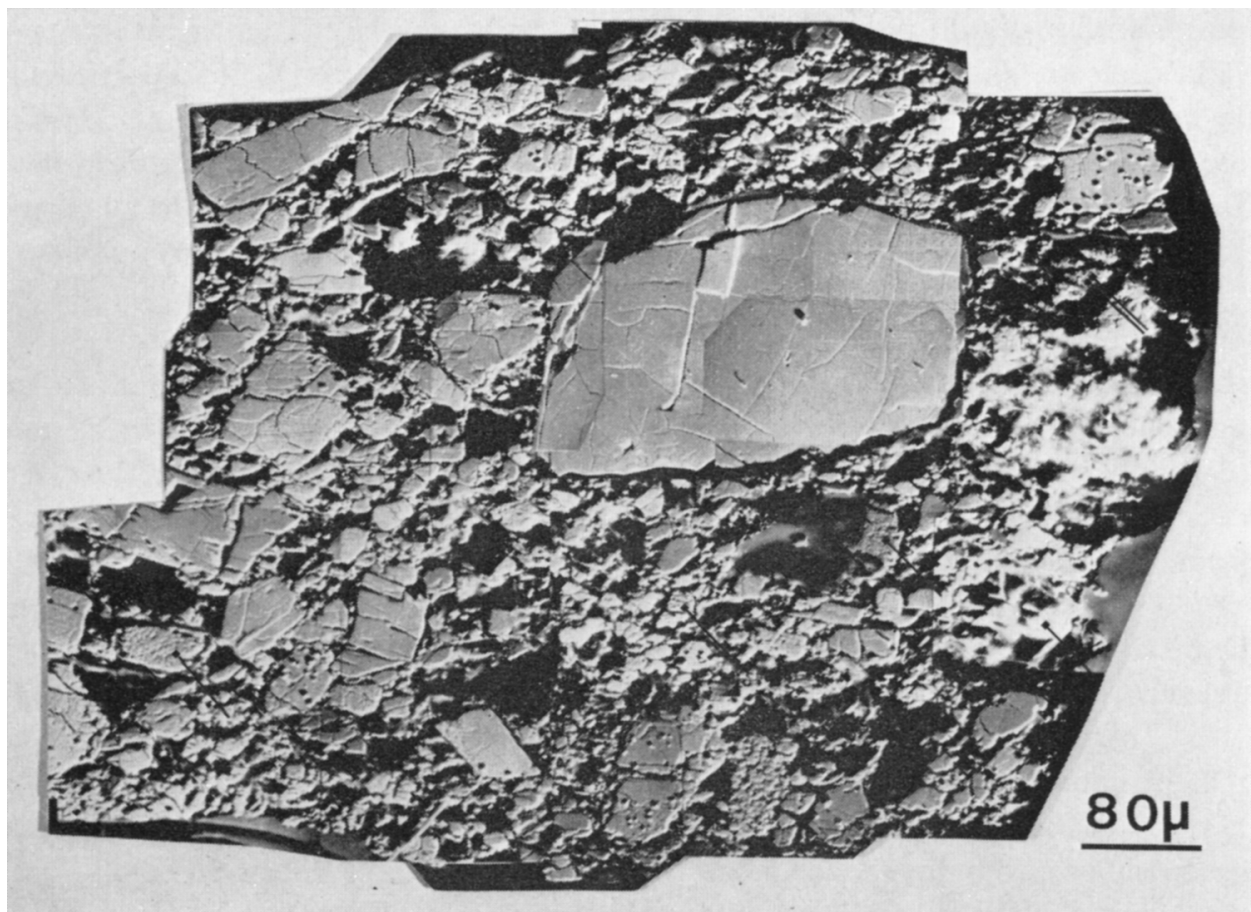

Fig. 2. Scanning electron micrograph of a polished section from a dark part of the Kapoyeta meteorite. This section was irradiated with fission fragments and etched $20 \mathrm{~min}$ in a boiling solution of sodium hydroxyde. Fission tracks and a high density of fossil tracks can be observed in the grains identified by single and double arrows respectively.

tracks in the micron-sized fragments by using transmission electron microscopy in order to minimize the possibility of missing the irradiation record in the smallest grains (we further applied this technique to examine grains from peculiar small parts in the dark parts including 'very dark' and 'white cloudy' parts, etc. ...);

(2) track zonings are frequently not related to a beam attenuation in the pyroxenes. Indeed we have observed fossil zonings in grains extracted from the deep interior of several igneous lunar rocks (and therefore well shielded from any $\mathrm{VH}$ solar flare irradiation) and also artificial zonings when studying the sensitivity for track registration in pyroxene and feldspar crystals. In this experiment [12] the fossil tracks were first erased by heating. Then the grains were polished, covered with a thin iron foil and exposed to artificial fluxes of $156 \mathrm{MeV}$ protons and $\alpha$-particles. The registration of the tracks of the recoil nuclei induced during the nuclear interactions of the incident particles with the iron nuclei was subsequently studied in the grains. The feldspars did not register these low energy iron recoil nuclei but out of about 20 pyroxenes grains we observed one grain with a high track density homogeneously distributed in the volume of the crystal (Figure $3 a$ ) and 2 grains with an edge zoned track distribution, inhomogeneous both along the edge of the grains and in its depth extension 


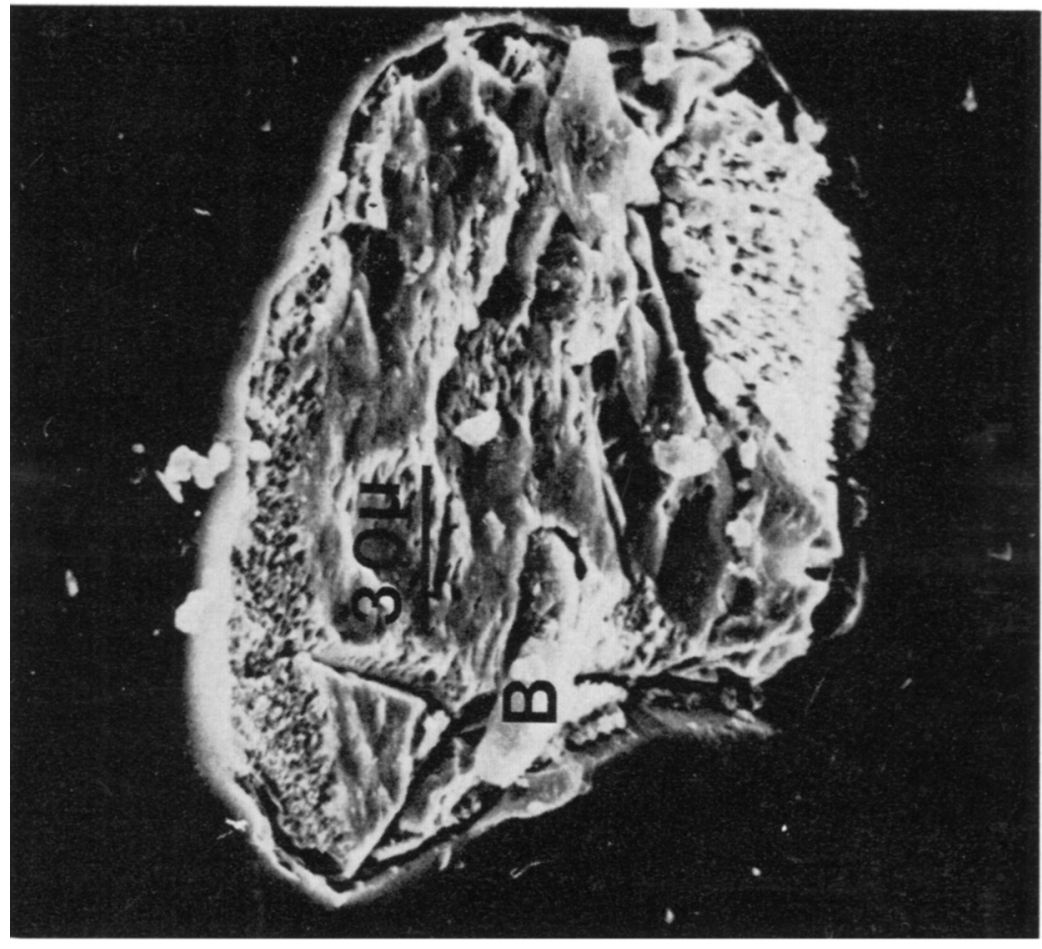

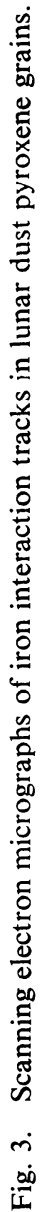

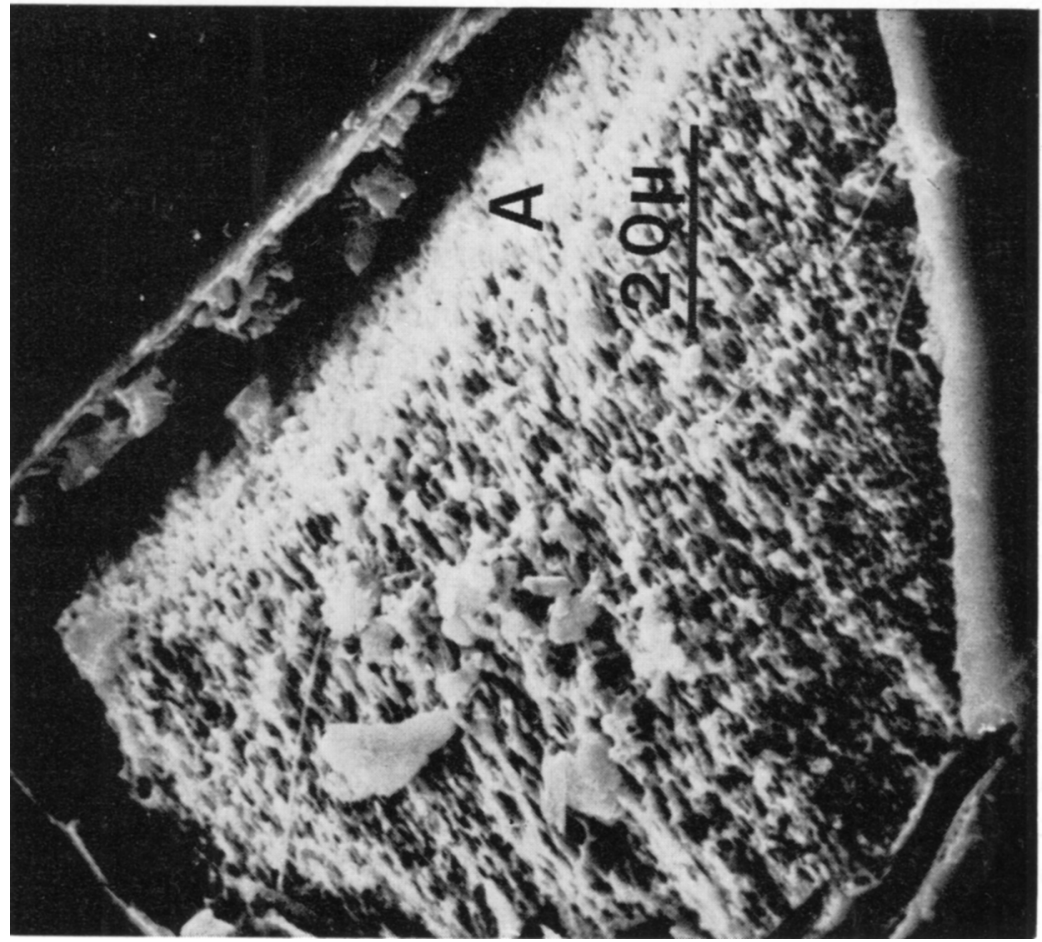


(Figure 3b). We reproduced the same results for the pyroxenes by using higher $Z$ material and $3 \mathrm{GeV}$ protons. For the feldspars irradiated in the same higher $Z$ conditions a very homogeneous track distribution was generally observed. We interpreted these striking and disturbing results as being due to a chemical zoning in the pyroxene grains modifying either their sensitivity for track registration or their etching rate. Therefore it is quite evident that feldspar crystals have to be selected in priority for identifying an 'individual' irradiation of meteoritic grains in space on a track gradient basis. If they are too rare or too small sized for an easy study then the pyroxenes could be used as a second choice material, but with great care especially if they contain iron as a major constituent. If the pyroxene zonings are unfrequent and inhomogeneous then an 'iron converter foil' experiment should be conducted to verify if the artificial zoning is not superimposed on the fossil zoning, otherwise the fossil tracks could te due to fossil recoils produced during the nuclear interactions of the galactic cosmic rays with medium heavy elements in the grains, after their 'compaction' into the meteorites.

We also developed [13] an additional criterion based on the transformation of the fossil irradiation record made of tracks and rare gas atoms when the lunar dust grains get either artificially heated or nuturally sintered into lunar breccias. In these grains the tracks are replaced by inclusions which grow and do not disappear when the annealing temperature is increased and which are observable by transmission electron microscopy (Figure 5). Therefore we searched also for inclusions in the 'sintered' grains from the dark parts of each of the gas-rich meteorites.

In the next chapter we will report our first and incomplete search for gas-rich meteorite crystals 'individually' irradiated in space and we will describe in more detail how we minimized various experimental uncertainties.

\section{The Ultramicroscopic Irradiation Record from Lunar Dust Particles to Solar Type Gas-Rich Meteorites}

\section{A. LUNAR DUST GRAINS}

Our transmission and scanning electron microscope observations are given in more detail and exploited for other purposes elsewhere [13, 14]. The main features of the irradiation record are:

(1) in the finest and uncrushed crystalline dust grains a high proportion $(\sim 80 \%)$ of the grains contain a very high density of nuclear particle tracks exceeding frequently $10^{10} \mathrm{tracks} \cdot \mathrm{cm}^{-2}$;

(2) in the coarser dust grains the high track density seems to be registered in a superficial micron-sized layer; at depth $\gg 1 \mu$ the track density drops to much lower values in the range $10^{8}-10^{9} \operatorname{tracks} \cdot \mathrm{cm}^{-2}[13,14]$;

(3) with the SEM a surprisingly low proportion of the coarser grains show edge zoned track distributions which even in the most favorable case (Figure 4) are not completely homogeneous;

(4) when the track bearing grains are heated in a furnace they get loaded with 


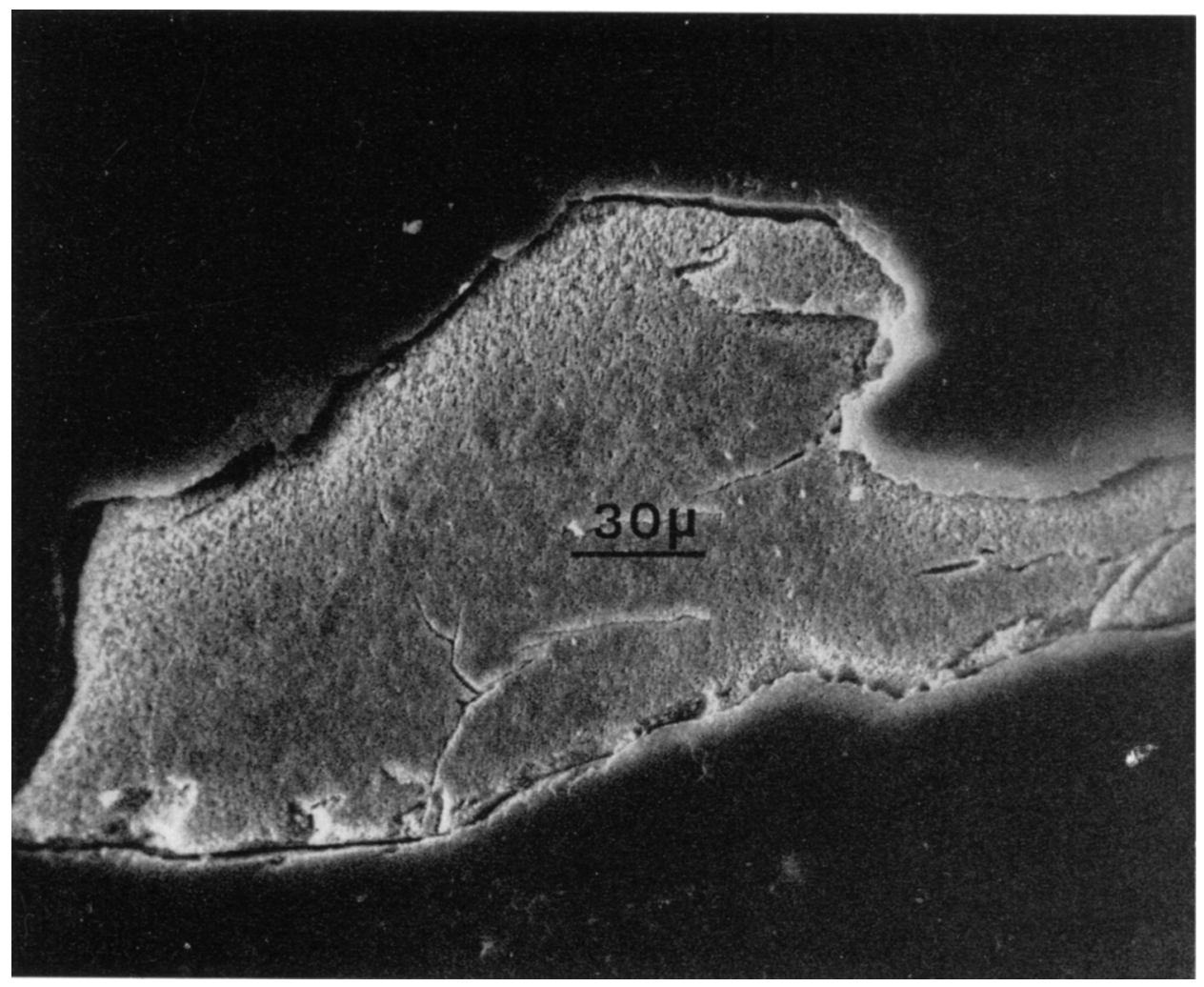

Fig. 4. Scanning electron micrograph of the best fossil zoning so far observed, in a lunar dust pyroxene grain.

high density of inclusions observable by high voltage electron microscopy (Figure 5a) and looking like the radiation induced phase transformations observed by Price and Walker [15] in their much earlier observation of artificially anealed fission fragment tracks in terrestrial mica;

(5) after a slight etching first developped by Barber et al. [16] short etched tracks are observed in the grains by $100 \mathrm{keV}$ electron microscopy.

\section{B. LUNAR BRECCIAS 10046 AND 10059}

With the $100 \mathrm{keV}$ and $1000 \mathrm{keV}$ electron microscopes we already reported [7, 13] the following observations for the crystalline grains:

(1) a small proportion of the grains $(\sim 1 \%)$ contain high densities of latent tracks before etching;

(2) the grains are frequently loaded with fossil inclusions (Figure 5b);

(3) a small proportion of the grains $(\sim 10 \%)$ show high densities of etched nuclear particle tracks with the $100 \mathrm{keV}$ electron microscope. 


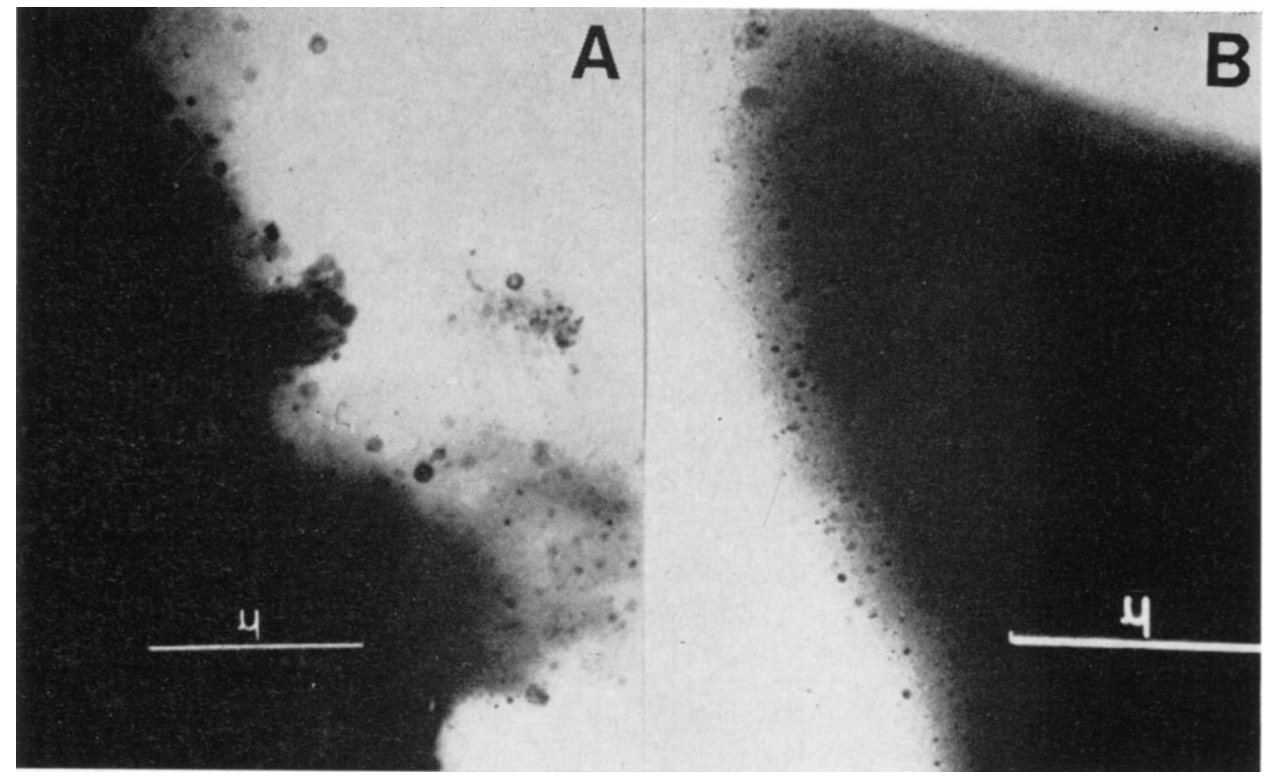

Fig. 5. $1 \mathrm{MeV}$ dark field micrographs of lunar dust grains. In B the grain was extracted from lunar breccia 10046 and has been 'naturally' metamorphized; in A the crystal was artificially heated at $800^{\circ} \mathrm{C}$, during $1 \mathrm{hr}$, under vacuum.

The SEM observation of etched polished sections reveals 3 different groups of grains:

(1) in about $1 \%$ of the grains with size smaller than about $30 \mu$ a reaction layer, probably indicative of high track densities registered in the whole volume of the grains, is observed (Figure 6a);

(2) all the largest glassy objects representing about $20 \%$ of the coarser grains contain very well formed etch pits attributable to nuclear particle tracks with densities up to $10^{8}$ tracks. $\mathrm{cm}^{-2}$ (Figure 6b);

(3) about $10 \%$ of the crystalline grains show shallow etch pits (Figure 6c) with densities up to $\sim 10^{9}$ tracks $\cdot \mathrm{cm}^{-2}$ and looking strikingly like those resulting from partially annealed VH tracks or from short spallation recoil tracks; by looking at more than 100 such grains in breccia 10046 we have not observed a track gradient similar to that reported in Figure 4.

\section{DARK PARTS OF SOLAR* TYPE GAS-RICH METEORITES}

For each meteorite (Weston, Pantar I, Breitschied, Kapoyeta), we first observed by high voltage electron microscopy, about 100 grains obtained by crushing small fragments hand picked in the dark parts of the meteorites. Then we etched $\sim 500$ grains in each crushed residue to observe slightly etched tracks by $100 \mathrm{keV}$ electron

* We also studied 'planetary' type gas-rich meteorites (Orgueil [8, 9], Mighei, Murray, Renazzo, Mokora) but this work will be reported later on [ $\left.{ }^{17}\right]$. 


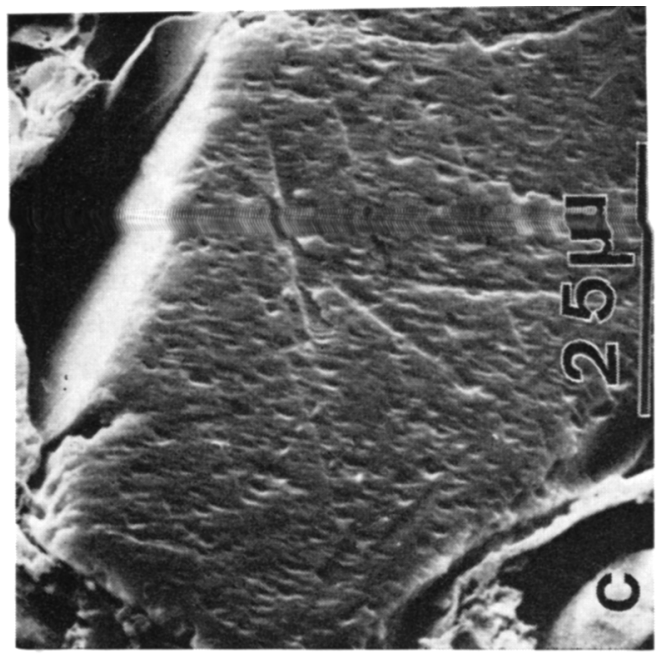

号

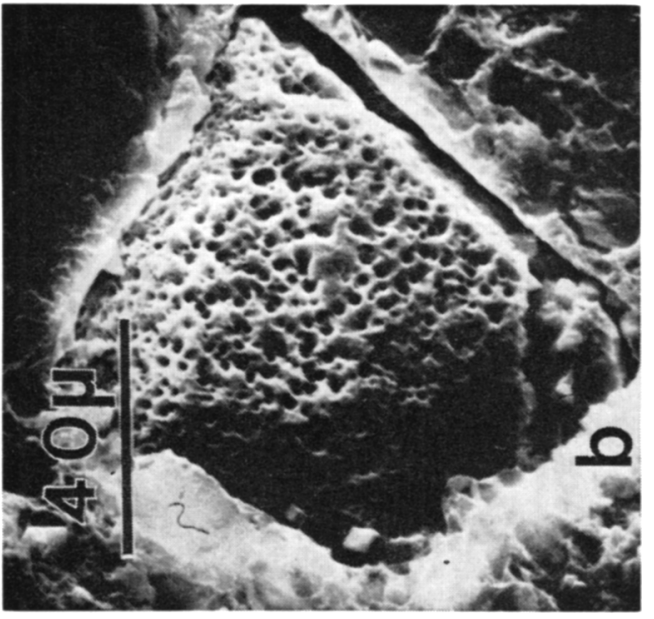

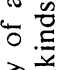

突宾

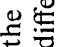

$\infty 0 \mathrm{~m}$

焉

공

몽

졿

06

옹

究

엉

․ㅗㄹ

은

芯

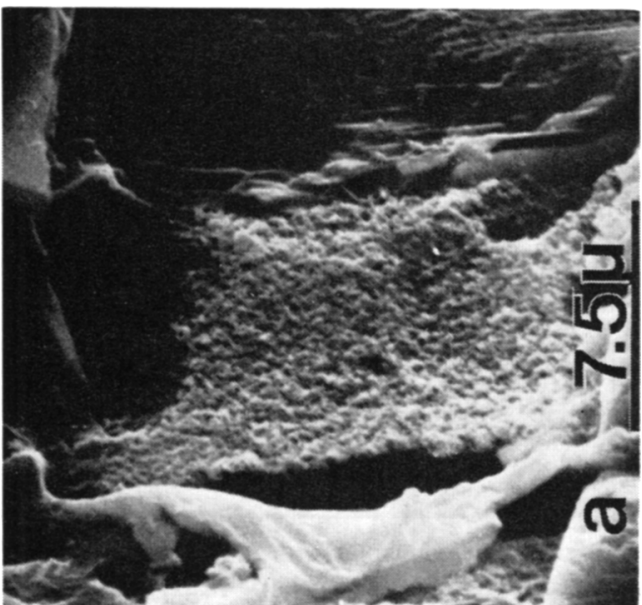

on

预

6

品 
microscopy in the same meteorites. Finally we studied an etched polished section of Kapoyeta with the SEM.

With the $1 \mathrm{MeV}$ electron microscope we did not observe either grains with high $\varrho$ values or inclusions similar to those reported in Figure 5a. Therefore the proportion of grains with high track density was certainly smaller than $1 \%$. Furthermore the grains showed an extensive microfracturing which was less frequent in the light parts of the meteorites.

With the $100 \mathrm{keV}$ electron microscope we discovered etched tracks only in Pantar I, where 2 grains showed etched track densities of about $2 \times 10^{9}$ and $6 \times 10^{9}$ tracks $\cdot \mathrm{cm}^{-2}$; but these tracks were certainly much longer than those constituting the high track density pattern in lunar dust grains and are likely of a different origin. Furthermore the striking microfracturing was beautifully revealed by such an etching (Figure 1)-on some area of this figure the microcracks could be easily confused with nuclear particle tracks as they are similarly etched but we have checked that contrarily to the tracks they are not annealed by heating the grains in a furnace.

With the SEM we observed Kapoyeta, because hand picked grains were extensively studied with this instrument by other groups $[4,5,6]$. We first irradiated the polished sections with a dose of fission fragment tracks of about $10^{7}$ tracks $\cdot \mathrm{cm}^{-2}$, well below the level expected from a solar flare irradiation. Then we etched for the feldspars

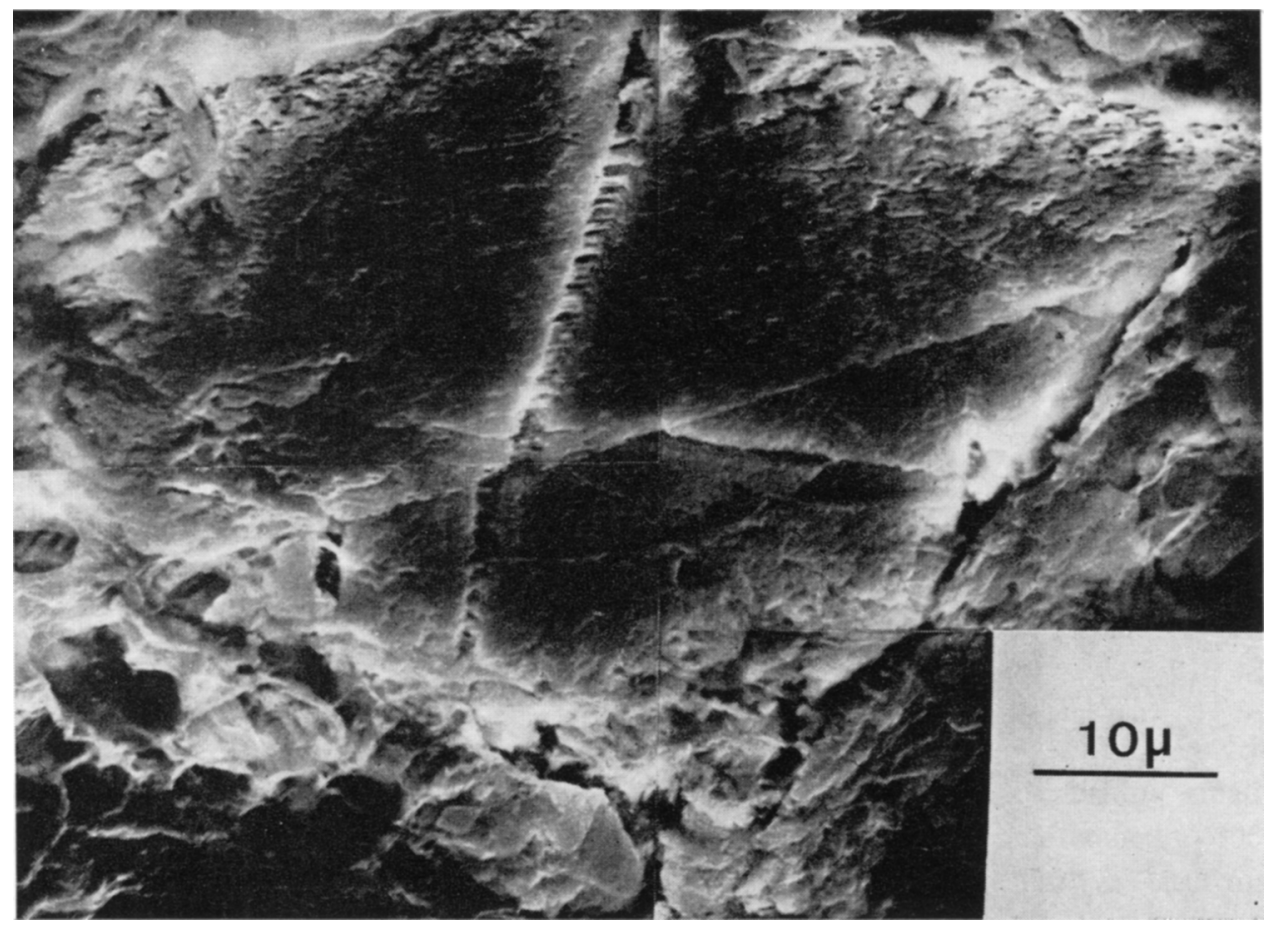

Fig. 7. Scanning electron micrograph of an inhomogeneous fossil track edge zoning in a pyroxene crystal extracted from a dark part of Kapoyeta. 
and determined the ratio between the number of grains showing track densities in excess of $10^{8}$ tracks $\cdot \mathrm{cm}^{-2}$ and that of grains with fission tracks. In Figure 2 this proportion is about $\frac{1}{7}$ but indeed by scanning a larger area we determined that it was smaller than $\frac{1}{30}$. Then we studied the possible track gradients and microprobed the grains with high $\varrho$ values to check further their nature, looking in particular for $\mathrm{P}$ and $\mathrm{Zr}$ which are generally considered as tracer for high uranium bearing phases [11]. Finally we repeated these observations-but we also microprobed for iron-for the pyroxenes which were much more abundant and much larger than the feldspars (in Figure 2 the pyroxenes are essentially all the grains without fission tracks). Our very priliminary results are: (1) out of 30 feldspar grains only one had a $\varrho$ value $\gtrsim 10^{8}$ tracks $\cdot \mathrm{cm}^{-2}$ but $n o$ gradient was observed; (2) out of 63 pyroxene grains 14 had $\varrho$ values in excess of $10^{8}$ tracks $\cdot \mathrm{cm}^{-2}$ but only one grain contained a fossil zoning (Figure 7) which was very inhomogeneous; (3) the tracks in the pyroxenes were generally much shorter than the fission fragments tracks simultaneously etched in the grains.

Very recently we also conducted a much faster survey of a Kapoyeta polished section etched for pyroxene grains and already studied with the SEM, by using Nomarski phase contrast in reflected light with an optical microscope. This survey confirmed very clearly the SEM observations thus showing that reflected light optical microscopy can be first used to unravel the irradiation record of gas-rich meteorite in identifying the high $\varrho$ grains to be further studied with the SEM.

\section{Discussion}

\section{A. COMPARISON OF THE PRESENT RESULTS TO THOSE OF OTHER WORKERS}

Our results with gas-rich meteorites disagree either with previous works concerning the study of etched tracks with the SEM in hand-picked grains $[4,5,6]$ or with the transmission electron microscope observations of Barber et al. [3] reported later than our already unsuccessful preliminary attempt [8] to find very high $\varrho$ values in gas-rich meteorites with a high voltage electron microscope: contrarily to the other groups, we have no clear evidence from track studies alone, for an 'individual irradiation' of the constituent grains of the dark parts of solar type gas-rich meteorites. Therefore we want strongly to point out that the following discussion has to be considered as a very preliminary one as long as the discrepancies between the different groups will not be understood.

These main discrepancies are:

(1) contrarily to the groups of Lal and Pellas which report by oral communications the existence of feldspar grains with homogeneous track gradients in Kapoyeta, we still have not discovered one feldspar grain with a gradient in this meteorite by using a more carefully experimental approach which can handle the very small sized feldspar grains appearing in Figure 2 without the uncertainties attached to any hand picking technique;

(2) Pellas reported a high proportion $(\sim 30 \%)$ of 'irradiated' pyroxene grains in 
Kapoyeta. This proportion only agrees with that of about $20 \%$ determined in the present work for pyroxene grains showing high $\varrho$ values $\left(\gtrsim 10^{8} \operatorname{tracks} \cdot \mathrm{cm}^{-2}\right)$. But unfortunately the proportion of these high $\varrho$ grains showing a track gradient was much smaller $(\sim 2 \%)$ and all the gradients we so far studied were quite inhomogeneous both along the edge of the grains and in their depth extension;

(3) in the high $\varrho$ pyroxenes we observed generally short and not long tracks;

(4) from their transmission electron microscope observations, Barber et al. [3] seem to have foud a lot of very high $\varrho$ grains in Fayetteville and Kapoyeta as they said "Although the meteorite studies are still at a preliminary stage, our observation of track densities comparable to those in lunar grains and at least 20 times greater than had been originally reported in track-rich meteorite grains [4, 5] are highly significant because they remove the previous major distinction [8] between lunar grains and meteoritic grains and suggest the possibility of a similar origin". However our earlier [8] and present observations do not verify such a statement because we find that the proportion of grains with latent track densities in excess of $10^{10}$ tracks $\cdot \mathrm{cm}^{-2}$ is certainly much smaller $(<1 \%)$ than in the lunar dust particles and that the proportion of grains with high densities of slightly etched tracks is $\sim 1 \%$ in the most favorable case of Pantar I where the maximum $\varrho$ value is still much smaller than those in the lunar dust grains.

Several tentative explanations for the discrepancies can be proposed:

(1) it is quite certain that a major difficulty in determining a proportion of grains with a gradient of etched tracks in the highly fractured gas-rich meteorites is to take really into account all the single grains in which the etching could have revealed the tracks and not for example count 5 grains for one highly fractured grain. We minimized this difficulty by preirradiating the polished sections with fission fragments and by performing microprobe and back scattered electron works with the same apparatus on the same area. The statistics of the Lal and Pellas groups are certainly different from ours because by hand picking they selected the largest crystals and we presume that they did not take into consideration in their statistics the more abundant smaller grains in their 'gently' crushed residue;

(2) our reported lack of grains with an homogeneous edge zoned track distribution is not surprising because such grains are very rare even in those extracted from the lunar dust and breccias. Therefore contrarily to the other investigators we did not expect to find a better zoning in gas-rich meteorites except if the constituent grains of these meteorites get processed by irradiation, erosion or 'sintering' mechanisms totally different from those acting in the lunar regolith;

(3) the discrepancies between our results and those of Barber et al. [3] are much more difficult to understand. Indeed by comparing our high voltage dark field micrographs of lunar dust grains loaded with latent tracks it was quite evident that these tracks were much easily observed with our $1 \mathrm{MeV}$ microscope than with their 650 $\mathrm{keV}$ microscope generally operated at $500 \mathrm{keV}$. One of the explanation for the better contrast and resolution was the smaller chromatic aberration at $1 \mathrm{MeV}$. As a consequence our estimate of the proportions of lunar dust crystalline grains loaded with 
latent tracks was generally greater than theirs. Therefore it seems unlikely that 'suddenly' we would have missed the high latent track densities in the gas-rich meteorite grains. Another possibility is that the Berkeley group has foud a still unreported criterion to detect irradiated grains by a quick survey of polished sections?

\section{B. FABRIC OF THE SOLAR TYPE GAS-RICH METEORITES AND LUNAR BRECCIAS}

The track irradiation record in gas-rich meteorites is probably different from that in other types of meteorites [1]. However we think that at the present time this record is not sufficiently well understood and is too far from the ideal record expected from grains individually exposed to energetic solar $\mathrm{VH}$ nuclei to conclude from track evidence alone that the dark parts of the solar type gas-rich meteorites have been formed by the 'sintering' of grains individually irradiated in space. Feldspar grains which are quite rare in the gas-rich meteorites have to be systematically studied with the technique we described and the beam attenuation origin for any possible zoning has to be checked each time with a converter foil experiment. Our results also cast serious doubts upon 2 earlier conclusions by Pellas et al. [5]: (1) the grains have certainly not 'been irradiated all around' and therefore it is no longer necessary to argue that the irradiation took place when the grains were freely floating in space at an early stage in the solar system history; (2) the distorsion appearing on X-rays diffraction patterns is not due to radiation damage unobserved by transmission electron microscopy but most likely to the extensive microfracturing in the grains.

The main evidence still supporting this hypothesis of a 'premeteoritic' irradiation is based on the great superficial enrichment of the dark part grains in rare-gas with abundance patterns and isotopic ratios similar to those produced by a solar wind implantation in aluminum foils deployed on the Moon surface during the Apollo 11 and 12 missions and subsequently brought back on the Earth [18]. However it should be definitively checked if the high densities of etchable 'track like' microfractures in the grains could not interfere with the conclusion-based on etched rare-gas release experiments [19]-that the rare-gas atoms are implanted in the most superficial layer of the grains.

Mason and Melson [20] have recently suggested that some solar type gas-rich meteorites are breccias which have been less metamorphized than lunar breccias. It will certainly be fruitful to check further the validity of this hypothesis by correlating the most striking texture features we observed in the dark parts (the extensive microfracturing and the absence of amorphous or highly disordered grains) to the following observations: (1) the microfracturing is less marked but still observable in the light parts of the same meteorites; (2) the lunar dust material most likely processed by micrometeorite and meteorite impacts appears as a two component matrix made of a totally amorphous minor fraction mixed to a major fraction of well ordered crystals in which microfracturing is very unfrequent and these characteristics are also observed in lunar breccias; (3) in the lunar breccia grains a major proportion of the tracks have been erased and replaced by inclusions; (4) Shergotty which is considered as a typical example of non gas-rich shocked meteorite, contains $\sim 20 \%$ of highly 
disordered grains but the microfracturing is less extensive than that appearing in Figure 1.

The track irradiation record in the lunar breccias so far studied has probably been 'metamorphized' by a heat sintering of the grains. This conclusion is supported by the observation of 'thermal like' inclusions and by the marked decrease in the proportion of grains with etched and latent tracks. Therefore we hope that the present methods when applied to the Apollo 14 rocks which are mostly complex 'breccias within breccias' will greatly help in understanding their origin.

\section{CHARACTERISTICS OF ANCIENT SOLAR NUCLEAR PARTICLE FLUXES}

It seems to us that the optimistic view of considering that the irradiation record in the gas-rich meteorites will allow the study of ancient solar flare cosmic rays, very early in the solar system history $[4,5,6]$, is far from reach at the present time. We think that it is much easier to find and exploit a solar record in lunar material.

But we also pointed out [21] that good track gradients to conduct such studies are very rare in the grains extracted from the lunar dust and lunar breccias. Therefore it appears that the best method to determine the characteristics of 'ancient' solar flare cosmic rays is to use polished sections containing the 'top' surfaces of lunar igneous rocks and to study the depth variation of the track density in the feldspar grains observed in these sections, by using methods described in the work of Crozaz et al. [3].

However, if our preliminary transmission [13] and high resolution scanning electron microscope [14] observations are confirmed by further works, then a high proportion of the lunar dust grains also contain 2 other types of homogeneous solar irradiation records which can be potentially exploited by methods described elsewhere [13, 21$]$. These records are registered in the most superficial layers of the grains and would be due: (1) to a solar wind implantation producing a superficial metamictized layer looking like a coating surrounding the grains, with a thickness of about $500 \AA$; (2) to solar 'suprathermal' ions building up high track densities in excess of $10^{10}$ tracks. $\cdot \mathrm{cm}^{-2}$ in a micron-sized superficial layer in the grains.

\section{Acknowledgements}

All the transmission microscope observations were made by L. Durrieu and C. Jouret at the Institut d'Optique Electronique du CNRS, Toulouse, France. We acknowledge the generous help of the JEOL Company for the SEM observations. One of us (M. Maurette) is deeply indebted to Dr R. Bernas for his very active and enthusiastic support and interest. We also thank Drs R. Hutchinson (British Museum, London), B. Mason (Smithsonian Institution, Washington D.C.) and A. Widatalla (Geological Survey Dept., Sudan) for the loan of meteoritic samples. We wish to express our gratitude to B. Vassent for her efficient and clever assistance with the experimental work and to J. Caro for her help in the manuscript preparation. 


\section{References}

[1] Walker, R. M.: 1972, 'Fossil Track Studies in Extraterrestrial Materials', Rad. Effects (in press).

[2] Suess, H. E., Wanke, H., and Wlotzka, F.: 1964, Geochim. Cosmochim. Acta 28, 595.

[3] We only give the references of the most recent contributions in press in Geochim. Cosmochim. Acta (1971):

Arrhenius, G., Liang, S., Mac Dougall, D., Wilkening, L., Bhandari, N., Bhatt, S., Lal, D., Rajagopalan, G., Tamhane, A. S., and Venkatavaradan, V. S.: 'The Exposure History of the Apollo 12 Regolith'.

Barber, D. G., Cowsik, R., Hutcheon, I. D., Price, P. B., and Rajan, R. S.: 'Solar Flares, the Lunar Surface and Gas-Rich Meteorites'.

Borg, J., Durrieu, L., Jouret, C., and Maurette, M.: 'Ultramicroscopic Features in MicronSized Lunar Dust Grains and Cosmophysics'.

Crozaz, G., Wa'ker, R. M., and Woolum, D.: 'Nuclear Track Studies of Dynamic Surface Processes on the Moon and the Constancy of Solar Activity'.

Fleischer, R. L., Hart, H. R., and Comstock, G. M.: 'Very Heavy Solar Cosmic Rays: Energy Spectrum and Implication for Lunar Erosion'.

[4] Lal, D. and Rajan, R. S.: 1969, Nature 223, 269.

[5] Pellas, P., Poupeau, G., Lorin, J. C., Reeves, H., and Audouze, J.: 1969, Nature 223.

[6] Lorin, J. C.: 1969, J. Physique 30, C3-102.

[7] Durrieu, L., Jouret, C., Leroulley, J., and Maurette, M.: 1971, Jernkontorets Ann., in press.

[8] Borg, J., Dran, J. C., Durrieu, L., Jouret, C., and Maurette, M.: 1970, Earth Planetary Sci. Letters 8, 379.

[9] Dran, J. C., Durrieu, L., and Jouret, C.: 1970, Earth Planetary Sci. Letters 9, 391.

[10] Fleischer, R. L.: 1968, Geochim. Cosmochim. Acta 32, 983.

[11] Burnett, D., Monnin, M., Seitz, M., Walker, R. M., and Yuhas, D.: 1971, Geochim. Cosmochim. Acta, in press.

[12] Bastin, G., Borg, J., Dran, J. C., Maurette, M., and Vassent, B.: 1970, Proceeding of the VIIth International Colloquium on Corpuscular Photography and visual solid detectors, Barcelona, July 1970

[13] See for example the work of Borg et al. in reference [8].

[14] Borg, J., and Vassent, B.: 1972, this volume, p. 298.

[15] Price, P. B. and Walker, R. M.: 1962, J. Appl. Phys. 33, 3410.

[16] Barber, D. J., Hutcheon, I., and Price, P. B.: 1971, Science 171, 372.

[17] Dran, J. C., Duraud, J. P., Durrieu, L., Jouret, C., Legressus, R., and Maurette, M.: 1971, to be submitted to Geochim. Cosmochim. Acta.

[18] Geiss, J., Eberhardt, P., Bühler, F., Meister, J., and Signer, P.: 1970, J. Geophys. Res. 75, 5972.

[19] Eberhardt, P., Geiss, J., and Groglern, N.: 1965, J. Geophys. Res. 70, 4375.

[20] Mason, B. and Melson, W.: 1970, The Lunar Rocks, Wiley-Interscience.

[21] Borg, J., Durrieu, L., Jouret, C., and Maurette, M.: Second Lunar Science Conference, unpublished proceedings. 